\title{
Serum procalcitonin in pregnancy-associated sepsis: A case control study
}

\author{
R Agarwal, ${ }^{1}$ MD (Obstetr Gynaecol), P Priyadarshini, ${ }^{1} \mathrm{MD}$ (Obstetr Gynaecol), M Mehndiratta, ${ }^{2} \mathrm{MD}$ (Biochemistry) \\ ${ }^{1}$ Department of Obstetrics and Gynaecology, Guru Teg Bahadur Hospital and University College of Medical Sciences, Delhi, India \\ ${ }^{2}$ Department of Biochemistry, Guru Teg Bahadur Hospital and University College of Medical Sciences, Delhi, India
}

Corresponding author: R Agarwal (rachna_anila@yahoo.co.in)

Background. Procalcitonin (PCT) is an established marker for sepsis, particularly bacterial, but its use in diagnosing pregnancy-associated sepsis (PAS) is relatively unexplored.

Objective. To investigate whether PCT could be used for the diagnosis of PAS in our study population, and what the appropriate values might be for the diagnosis.

Methods. The study included 40 pregnant, post-abortal and postpartum women with PAS, identified using systemic inflammatory response syndrome criteria. Patients with hypertensive disorders, premature rupture of membranes, severe trauma, liver cirrhosis and lung or thyroid malignancies were excluded. Forty healthy term pregnant women with sterile urine cultures were taken as controls. PCT levels were obtained at admission and statistically compared between the groups. Severity and culture positivity in PAS were also assessed.

Results. Severe PAS was present in 30 patients. Overall culture positivity was seen in $55 \%(n=22)$ of subjects. Culture-positive patients had higher PCT levels $(3.03 \mu \mathrm{g} / \mathrm{L})$ compared with the culture-negative $(1.77 \mu / \mathrm{L})$, though this did not reach statistical significance $(p=0.235)$. Mean (standard deviation) PCT in PAS patients was $2.46(0.56) \mu / \mathrm{L}$, while in healthy controls it was $0.091(0.019) \mu \mathrm{g} / \mathrm{L}$. This difference was statistically significant $(p<0.001)$. In severe PAS, PCT levels $(3.12(0.70) \mu \mathrm{g} / \mathrm{L})$ were significantly higher than in non-severe $(0.49(0.21) \mu \mathrm{g} / \mathrm{L})$. A cut-off of $0.125 \mu / \mathrm{L}$ gave the best diagnostic marker for PCT for PAS (sensitivity $67.5 \%$; specificity $82.5 \%$ ).

Conclusion. PCT is raised significantly in PAS, especially in culture-positive situations. A PCT level $>0.125 \mu \mathrm{g} / \mathrm{L}$ is suggested as the critical level for PAS diagnosis in appropriate clinical settings. Additionally, mean PCT levels differed significantly in severe v. non-severe PAS.

S Afr J Obs Gynae 2019;25(1):15-19. DOI:10.7196/SAJOG.2019.v25i1.1397

Procalcitonin (PCT) is considered to be the prohormone of the active compound calcitonin. ${ }^{[1]}$ PCT is produced in response to infection in association with inflammatory markers IL-1, TNF and IL-6, and strongly correlates with the extent and severity of bacterial infection. PCT can be detected in plasma as early as 2 hours after exposure to bacterial endotoxins. It increases over 3 - 4 hours, peaks at 6 hours and then plateaus for up to $20-72$ hours. PCT has gradually acquired a reputation for the early detection and estimation of the degree of inflammation, and for prognosis. ${ }^{[1]}$ PCT levels can also be used for the selection of antibiotics, and the evaluation of their efficacy. ${ }^{[1]}$

PCT estimation in pregnancy has focused mainly on the determination of critical levels during various stages, ${ }^{[2,3]}$ hypertensive pathologies, ${ }^{[4]}$ infection in preterm premature rupture of membranes ${ }^{[5-10]}$ or asymptomatic bacteriuria. ${ }^{[11]}$ One recent study investigated using PCT to detect frank sepsis in pregnancy, with encouraging results. ${ }^{[12]}$

The reported incidence of pregnancy-associated sepsis (PAS) in high-income countries ranges from 12 - 35 per 100000 deliveries, with accompanying maternal mortality ranging from 0.85 to 1.13 per $100000 .^{[13-15]}$ In low-income countries, PAS is one of the foremost causes of maternal mortality and morbidity. ${ }^{[16]} \mathrm{A}$ haematological investigation to aid in the early diagnosis of PAS would be an asset. We devised this prospective case control study to determine the PCT levels that could identify PAS and its severity.

\section{Methods}

The study was conducted between October 2016 and March 2018 in the obstetric emergency department of a tertiary-level health facility in a low-income country. Clearance from the institutional ethics committee of the University College of Medical Sciences, Delhi, India (certificate supplied; dated 17 October 2016), was provided, and prior consent obtained from all patients.

\section{Sample size estimation}

There were no previous studies to guide sample size calculations. Considering a PCT median (interquartile range) level of 2.29 $(0.72-9.07) \mu / \mathrm{L}$ in bacteraemic and $0.25(0.10-0.90) \mu / \mathrm{L}$ in a nonbacteraemic group, we calculated that with an a of $5 \%$ and $90 \%$ power, estimating a difference of $2 \mu / \mathrm{L}$, a sample size of 20 cases was required in each group. ${ }^{[17]}$ The present study enrolled 40 cases and 40 controls, to enhance quality and statistical strength. Because the study was conceived and initiated prior to the publication of the Sepsis-3 guidelines, older sepsis definitions were utilised. ${ }^{[18,19]}$

\section{Inclusion and exclusion criteria}

All pregnant, post-abortal ( $\leq 2$ weeks) and postpartum women ( $\leq 6$ weeks) with clinical sepsis were investigated using systemic inflammatory response syndrome sepsis criteria (mean arterial blood pressure $<65 \mathrm{mmHg}$; systolic blood pressure $\leq 90 \mathrm{mmHg}$; heart rate $\geq 110 / \mathrm{min}$; respiratory rate $\geq 22 / \mathrm{min}$; temperature $\geq 38^{\circ} \mathrm{C}$ 
or $\leq 36^{\circ} \mathrm{C}$; and leucocyte count $\geq 1.4 \times$ $10^{9} / \mathrm{L}$ or $<4 \times 10^{9} / \mathrm{L}$ ). Patients meeting $\geq 2$ of the above criteria were classified as having PAS, and enrolled in the study (Fig. 1). ${ }^{[18,19]}$ We excluded patients with known pathologies that could alter PCT: preeclampsia/eclampsia; premature rupture of membranes; severe trauma; liver cirrhosis; or lung or thyroid malignancies. Severe sepsis was defined as an infection related to one or more organ dysfunctions within 24 hours of admission. ${ }^{[20]}$ Healthy term pregnant women with sterile urine cultures were used as controls.

Detailed clinical and pertinent laboratory and imaging tests were performed for all enrolled patients. Blood, high vaginal swab and pus (if present) cultures were sent for bacterial culture and sensitivity. We considered the specimen culture positive if a single microbial growth was isolated in any of the above samples. PCT levels were obtained along with other haematological investigations at admission. For organ failure assessment, the following key body systems were assessed and monitored: pulmonary; cardiac; renal; hepatobiliary; and neurological. Patients were further managed as per hospital protocol and patient's condition.

\section{Method of PCT estimation}

Two-millilitre blood samples were collected from each subject in a plain vial for procalcitonin analysis. Serum was separated immediately and the samples stored in aliquots at $-80^{\circ} \mathrm{C}$. PCT analysis was performed by human PCT ELISA, which is a sandwich enzyme immunoassay for the quantitative measurement of human procalcitonin.

All descriptive parameters were expressed as percentages for qualitative parameters, and mean (standard deviation (SD)) for quantitative parameters. PCT levels were compared by non-parametric tests. Area under curve (AUC) graphs were plotted to show PCT diagnostic values for PAS and its severity.

\section{Results}

Our study sample comprised a young population with an age range of $20-30$ (mean (SD) 24.31 (4.63)) years. Of the 40 PAS patients, $22(55 \%)$ were post partum, 14 antenatal (35\%) and 4 post abortal (10\%). Severe sepsis was detected to be present in 30 of the subjects (75\%), of whom 17 (56.7\%), $10(33.3 \%)$ and $3(1 \%)$ patients were post partum, antepartum and

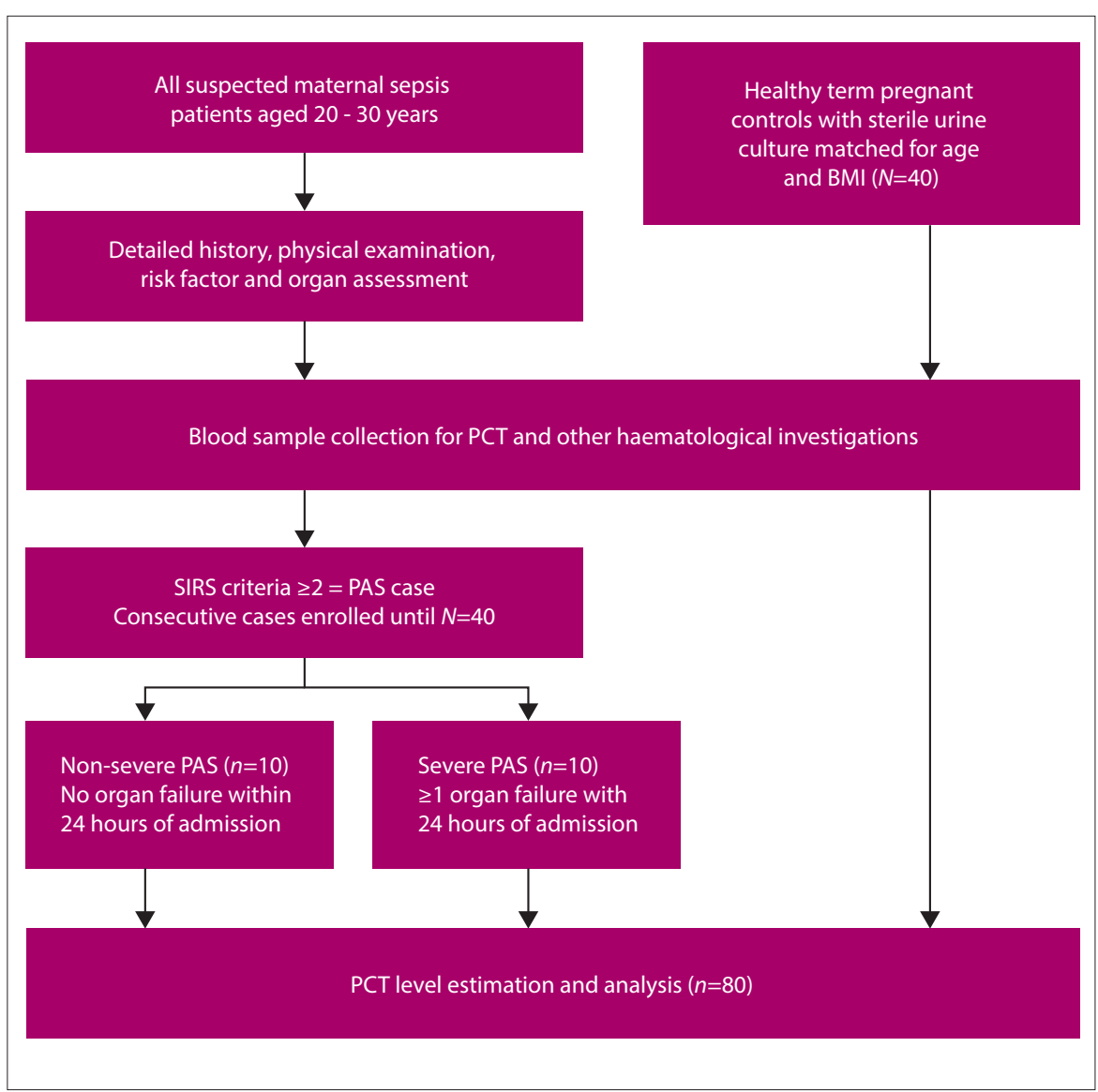

Fig. 1. Study design. $(B M I=$ body mass index $; P C T=$ procalcitonin $;$ SIRS $=$ systemic inflammatory response syndrome.)

post abortal, respectively. The distribution of various clinical and laboratory parameters in the study subjects is detailed in Table 1.

\section{Culture positivity and PCT levels}

Genital infection (high vaginal swab culture positive) was seen in $27.5 \%$ of PAS patients, followed by urinary tract infection (urine culture positive) in $20 \%$ and septicaemia with blood culture positive in $17.5 \%$ of cases (Table 2). Overall culture positivity (any culture positive) was seen in 22 (55\%) subjects with PAS. The most common organism identified in positive blood and genital cultures was Staphylococcus aureus. The PCT levels in culture-positive subjects were higher (mean (SD) 3.03 $(0.80) \mu / L)$ compared with culture-negative patients $(1.77(0.74) \mu / L)$, but this was not statistically significant $(p=0.235)$.

\section{PAS cases v. controls}

Serum PCT in the PAS cases was in the range of $0.01-10.07 \mu / \mathrm{L}$, while in healthy controls it was $0.01-0.56 \mu / \mathrm{L}$. The mean
(SD) PCT level in the cases was 2.46 $(0.56) \mu / L$, while in the controls it was $0.091(0.019) \mu / \mathrm{L}(p<0.001)$. On receiver operating characteristic (ROC) analysis, AUC was 0.792 , with a cut-off of $0.125 \mu / \mathrm{L}$ that gave the best combination of sensitivity (67.5\%) and specificity (82.5\%) (Fig. 2).

\section{Severe v. non severe PAS}

The mean (SD) PCT level in severe PAS cases was $3.12(0.70) \mu / \mathrm{L}$, while in nonsevere it was $0.49(0.21) \mu / \mathrm{L} \quad(p=0.04)$. On ROC analysis, the seemingly most appropriate cut-off value of PCT between severe and non-severe was $1.505 \mu / \mathrm{L}$ (sensitivity $46 \%$; specificity $90 \%$; statistically insignificant) (Fig. 3).

\section{Discussion}

PAS is associated with considerable morbidity and mortality, and therefore its early detection is of the utmost importance in obstetrics. A major dilemma faced, however, is that sepsis during pregnancy is not reliably predicted by commonly used laboratory tests. Clinical signs in PAS, such as fever and tachycardia, may at times 


\section{RESEARCH}

Table 1. Parameters in PAS group

\begin{tabular}{|c|c|c|c|}
\hline Parameter & $\begin{array}{l}\text { All PAS } \\
(N=40)\end{array}$ & $\begin{array}{l}\text { Non-severe PAS } \\
(n=20)\end{array}$ & $\begin{array}{l}\text { Severe PAS } \\
(n=20)\end{array}$ \\
\hline \multicolumn{4}{|l|}{ Heart rate (bpm), $n(\%)$} \\
\hline$\geq 110(\%)$ & $37(92.5 \%)$ & 7 & 30 \\
\hline Normal & $3(7.5 \%)$ & 3 & 0 \\
\hline \multicolumn{4}{|l|}{ Total leucocytes count $\left(\times 10^{\%} / \mathrm{L}\right), n(\%)$} \\
\hline$\geq 1.4$ & $33(82.5 \%)$ & 7 & 26 \\
\hline$<4$ & $2(5 \%)$ & 0 & 2 \\
\hline Normal & $5(12.5 \%)$ & 3 & 2 \\
\hline \multicolumn{4}{|l|}{ Temperature $\left({ }^{\circ} \mathrm{C}\right), n(\%)$} \\
\hline$\geq 38^{\circ} \mathrm{C}$ & $22(55 \%)$ & 6 & 16 \\
\hline$\leq 36^{\circ} \mathrm{C}$ & $3(7.5 \%)$ & 0 & 3 \\
\hline Normal & $15(37.5 \%)$ & 4 & 11 \\
\hline \multicolumn{4}{|l|}{ Respiratory rate (breaths per minute), $n$ (\%) } \\
\hline$\geq 22$ & $25(62.5 \%)$ & 6 & 19 \\
\hline Normal & $15(37.5 \%)$ & 4 & 11 \\
\hline \multicolumn{4}{|l|}{ Systolic blood pressure (mmHg), $n(\%)$} \\
\hline$\leq 90$ & $12(30 \%)$ & 2 & 10 \\
\hline Normal & $28(70 \%)$ & 8 & 20 \\
\hline \multicolumn{4}{|l|}{ Mean arterial pressure $(\mathrm{mmHg}), n(\%)$} \\
\hline$<65$ & $7(17.5 \%)$ & 1 & 6 \\
\hline Normal & $33(82.5 \%)$ & 9 & 24 \\
\hline Procalcitonin levels, mean $(\mathrm{SD})(\mu / \mathrm{L}$ (or $\mathrm{ng} / \mathrm{mL}))$ & $2.46(0.56)$ & $0.49(0.21)$ & $3.12(0.70)$ \\
\hline
\end{tabular}

\section{Table 2. Culture-positive PAS ( $n=22)$}

\begin{tabular}{ll}
\hline Organism involved & Culture positive, $n$ \\
\hline Septicaemia (blood culture positive), $n=7$ & \\
$\quad$ Staphylococcus aureus & 4 \\
MRSA & 3 \\
Genital infection (HVS positive), $n=11$ & \\
$\quad$ Staphylococcus aureus & 6 \\
Escherichia coli & 1 \\
MRSA & 2 \\
Acinobacter & 1 \\
Enterobacter & 1 \\
UTI (urine culture positive), $n=8$ & \\
Escherichia coli & 5 \\
Klebsiella pneumoniae & 1 \\
Candida albicans & 1 \\
Yeast & 1 \\
Wound infection (pus culture positive), $n=2$ & \\
Staphylococcus aureus & 1 \\
MRSA & 1
\end{tabular}

PAS = pregnancy-associated sepsis; HVS = high vaginal swab; MRSA = methicillin-resistant Staphylococcus aureus; UTI = urinary tract infection.

be unreliable. White blood cell counts and C-reactive protein can vary even in uncomplicated pregnancy. ${ }^{[21]}$ We therefore considered it worthwhile to investigate PCT use for the detection of sepsis in obstetric practice.

\section{PCT levels in healthy pregnant women}

Paccolat et al. ${ }^{[2]}$ studied 60 pregnant women without clinical infection, and determined the normal value ranges of PCT during the third trimester, at delivery and during the postpartum period. The median levels of PCT preterm ranged from 0.043 to

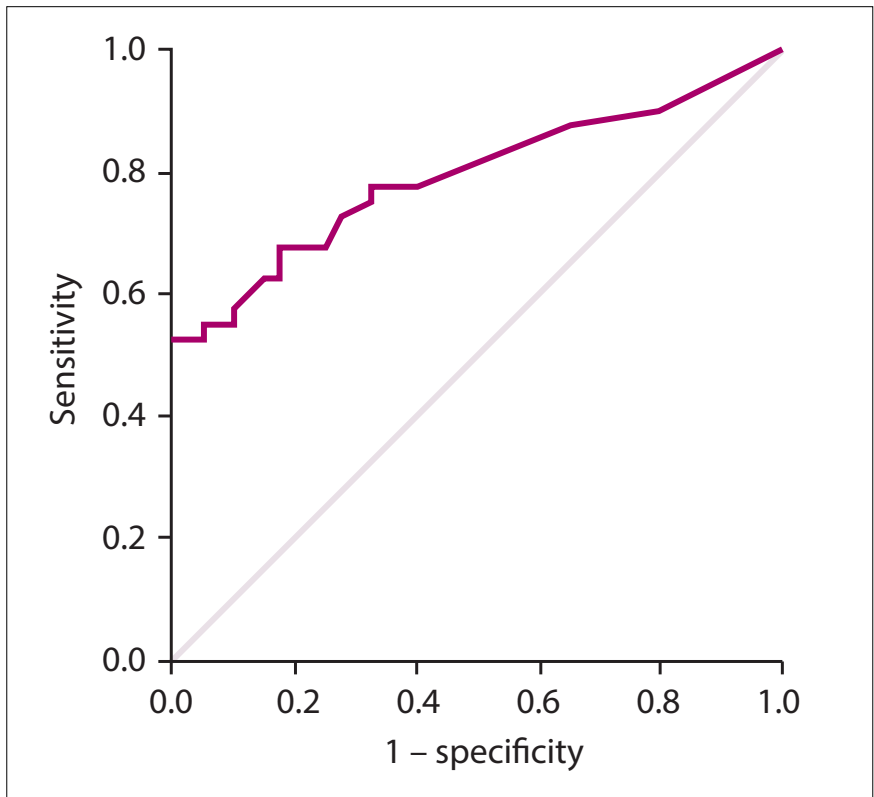

Fig. 2. ROC curve for cut-off procalcitonin levels in PAS cases $v$. healthy controls. $(R O C=$ receiver operating characteristic. $)$

$0.061 \mu / \mathrm{L}$ between 24 and 40 weeks, to $0.068 \mu / \mathrm{L}$ at delivery, and on postpartum days 2 - 10 from 0.200 to $0.060 \mu / \mathrm{L}$. The authors also specified a cut-off level of $0.25 \mu / \mathrm{L}$ (specificity $91 \%$ on day 3 postpartum) to rule out infection in the perinatal period. This is the same threshold is used to diagnose systemic infections and to guide the use of antibiotics in infected adult populations. ${ }^{[2]} \mathrm{Hu}$ et al. ${ }^{[3]}$ in a dedicated study of a healthy Chinese population, found reference intervals for PCT of $0.018-0.051 \mu / \mathrm{L}$ for the first and the second trimester, and $0.020-0.152 \mu / \mathrm{L}$ for the third trimester. Corresponding reference values for non-pregnant healthy women 


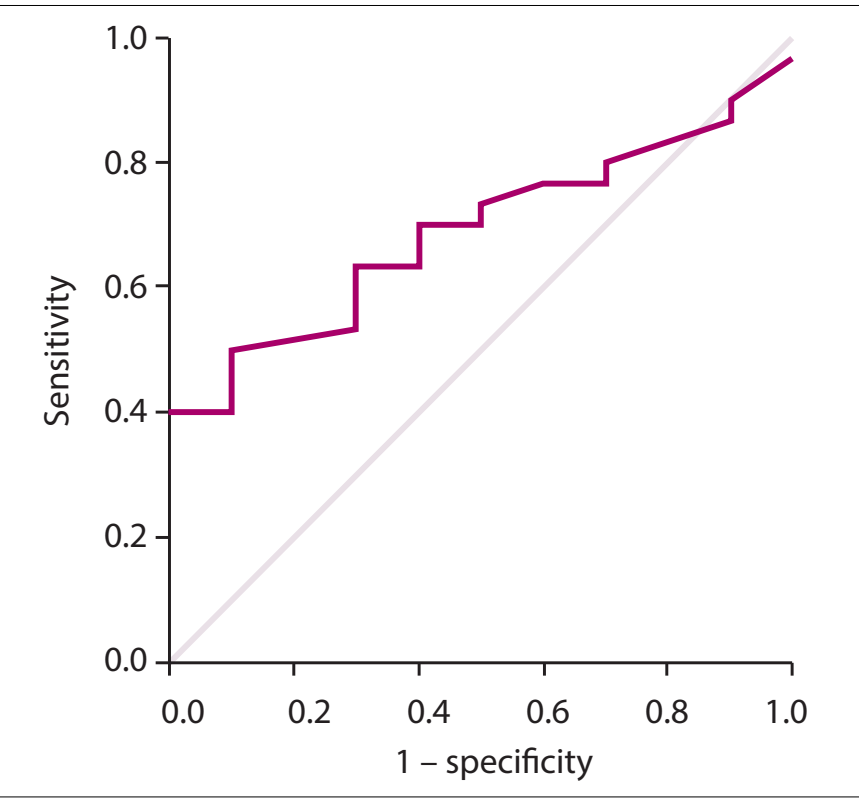

Fig. 3. ROC curve for cut-off procalcitonin levels in severe v. nonsevere PAS. (ROC = receiver operating characteristic.)

were $0.015-0.061 \mu / \mathrm{L}$. Our study revealed a higher PCT range of $0.01-0.56 \mu / \mathrm{L}$ in the third trimester of healthy pregnant controls than did the above studies.

\section{PCT in sepsis}

A Colombian study group conducted a cross-sectional study in 98 pregnant patients with suspected infection, and 45 with no infection. ${ }^{[12]}$ They found that mean (interquartile range (IQR) PCT levels were higher for the patients with bacterial infection $(0.09(0.05-0.022) \mu / L)$ than for non-infected patients $(0.05(0.025-0.06) \mu / \mathrm{L}) ; p<0.0001)$ and patients with non-bacterial infection $(0.05(0.05-0.07) \mu / L ; p=0.027)$. They postulated that the raised PCT levels acted as a strong marker for bacterial sepsis in pregnancy (AUC 0.73), and proposed a cut-off of $0.06 \mu / \mathrm{L}$ (sensitivity $60 \%$; specificity $84 \%$ ) for the diagnosis of bacterial sepsis.

The present study similarly found a significant difference between PCT levels in PAS cases and healthy controls. The mean value of PCT in PAS cases was $2.46 \mu / \mathrm{L}$, while in controls it was $0.091 \mu / \mathrm{L}$. Our study suggested that the best diagnostic potential for PCT is a cut-off of $0.125 \mu / \mathrm{L}$, at a sensitivity of $67.5 \%$ and specificity of $82.5 \%$. Additionally, mean PCT levels differed significantly in severe $(3.12 \mu / \mathrm{L})$ v. non-severe PAS $(0.49 \mu / \mathrm{L})(p=0.04)$. Although PCT levels were almost twice as high in the culture-positive PAS (mean $3.03 \mu / \mathrm{L}$ ) compared with the non-positive (mean $1.77 \mu / \mathrm{L}$ ) group, this difference did not reach statistical significance.

Because our original focus was on researching PCT levels in PAS, serial evaluations of PCT were not done, and antibiotic decisions were also not based on this marker. There were differences in the timing of collection of samples, which varied according to the patients' presentation to the emergency department. This may be one reason for our PCT diagnostic cut-off value being different from Paccolat et al.'s ${ }^{[2]}$ $(0.25 \mu / \mathrm{L})$ or Velasquez et al. ${ }^{[12]}(0.06 \mu / \mathrm{L})$. Another possible reason might be the greater percentage of severe PAS cases $(n=30 ; 75 \%)$ in our series, and therefore the higher PCT reference levels.

One strength of our study was the use of a matched cohort of pregnant young women, so that possible confounding factors for elevation of PCT were excluded. Patients with asymptomatic bacteriuria, pre-eclampsia/eclampsia or premature rupture of membranes were excluded. Besides having an overall robust sample size, this study also gathered information on PCT levels in PAS from the Asian continent, for the first time. Although pregnancy, with its complexities of physiological and hormonal changes, could pose significant challenges in determining the decisive PCT levels for diagnosing sepsis, we believe that their use should complement clinical findings. Our study contributes towards a threshold level of PCT for PAS, as there is no consensus at present. PCT levels may also be used to differentiate between non-severe and severe PAS. More studies are, however, warranted to precisely define the critical PCT levels, with obvious implications for monitoring and managing PAS.

\section{Conclusions}

We found that PCT was raised significantly in PAS, especially in culture-positive patients. A PCT cut-off of $0.125 \mu / \mathrm{L}$ (sensitivity $67.5 \%$; specificity $82.5 \%$ ) is suggested as the critical level for PAS diagnosis in appropriate clinical settings. Additionally, mean PCT levels differed significantly in severe v. non-severe PAS.

Acknowledgements. None.

Author contributions. Equal contributions.

Funding. None.

Conflicts of interest. None.

1. Wacker C, Prkno A, Brunkhorst FM, Schlattmann P. Procalcitonin as a diagnostic marker for sepsis: A systematic review and meta-analysis. Lancet Infect Dis 2013;13(5):426-435. https://doi. org/10.1016/S1473-3099(12)70323-7

2. Paccolat C, Harbarth S, Courvoisier D, Irion O, de Tejada BM. Procalcitonin levels during pregnancy, delivery and postpartum. J Perinat Med 2011;39(6):679-683. https://doi.org/10.1515/ jpm.2011.082

3. Hu Y, Yang M, Zhou Y, Ding Y, Xiang Z, Yu L. Establishment of reference intervals for procalcitonin in healthy pregnant women of Chinese population. Clin Biochem 2017;50(3):150-154. https://doi. org/10.1016/j.clinbiochem.2016.10.013

4. Duckworth S, Griffin M, Seed PT, et al. Diagnostic biomarkers in women with suspected preeclampsia in a prospective multicentre study. Obstet Gynecol 2016;128(2):245-252. https://doi org/10.1097/aog.0000000000001508

5. Torbé A. Maternal plasma procalcitonin concentrations in pregnancy complicated by preterm premature rupture of membranes. Mediators Inflamm 2007:35782. https://doi. org/10.1155/2007/35782

6. Torbé A, Czajka R. Are vaginal fluid procalcitonin levels useful for the prediction of subclinical infection in patients with preterm premature rupture of membranes? J Obstet Gynaecol Res 2005;31(5):464-470. https://doi.org/10.1111/j.1447-0756.2005.00321.x

7. Kuyumcuoglu U, Kangal K, Guzel AI, Celik Y. Clinical significance of procalcitonin in cervico-vaginal secretions of women with preterm rupture of membranes. Clin Exp Obstet Gynecol 2010;37(4):319-321.

8. Thornburg LL, Queenan R, Brandt-Griffith B, Pressman EK. Procalcitonin for prediction of chorioamnionitis in preterm premature rupture of membranes. J Matern Fetal Neonatal Med 2016;29(13):2056-2061. https://doi.org/10.3109/14767058.2015.1077224

9. Oludag T, Gode F, Caglayan E, Saatli B, Okyay RE, Altunyurt S. Value of maternal procalcitonin levels for predicting subclinical intra-amniotic infection in preterm premature rupture of membranes. J Obstet Gynaecol Res 2014;40(4):954-960. https://doi.org/10.1111/jog.12273

10. Ducarme G, Desroys du Roure F, Le Thuaut A, Grange J, Vital M, Dimet J. Efficacy of serum procalcitonin to predict spontaneous preterm birth in women with threatened preterm labour: A prospective observational study. BMC Pregnancy Childbirth 2018;18:65. https://doi.org/10.1186/ s12884-018-1696-2

11. Bilir F, Akdemir N, Ozden S, Cevrioglu AS, Bilir C. Increased serum procalcitonin levels in pregnant patients with asymptomatic bacteriuria. Ann Clin Microbiol Antimicrob 2013;12:25. https://doi. org/10.1186/1476-0711-12-25

12. Velasquez J, Zuleta J, Portilla P, Caicedo K, Portilla L, Patiño J. Usefulness of measuring procalcitonin (PTC) in pregnancy for the initial diagnosis of bacterial infection with systemic features. Am J Obstet Gynecol 2018;218(1):S514-S515. https://doi.org/10.1016/j.ajog.2017.11.401

13. Waterstone $\mathrm{M}$, Bewley $\mathrm{S}$, Wolfe $\mathrm{C}$. Incidence and predictors of severe obstetric morbidity: Case control study. BMJ 2001;322:1089-1093. https://doi.org/10.1136/bmj.322.7294.1089

14. Mabie WC, Barton JR, Sibai B. Septic shock in pregnancy. Obstet Gynecol 1997;90:553-561. https:// doi.org/10.1016/s0029-7844(97)00352-9

15. Centre for Maternal and Child Enquiries (CMACE). Saving Mothers' Lives: Reviewing maternal deaths to make motherhood safer: 2006 - 2008. The Eighth Report on Confidential Enquiries into Maternal Deaths in the United Kingdom. BJOG 2011;118(Suppl 1):S1-S203. https://doi. org/10.3410/f.9021958.9589054

16. Chhabra S, Kaipa A, Kakani A. Reduction in maternal mortality due to sepsis. J Obstet Gynaecol 2005;25(2):140-142. https://doi.org/10.1080/01443610500051346

17. Van Nieuwkoop C, Bonten TN, van't Wout JW, et al. Procalcitonin reflects bacteraemia and bacterial load in urosepsis syndrome: A prospective observational study. Crit Care 2010;14:R206. https://doi. org/10.1186/cc9328

18. Levy MM, Fink MP, Marshall JC. 2001 SCCM/ESICM/ACCP/ATS/SIS International Sepsis Definitions Conference. Crit Care Med 2003;31:1250-1256. https://doi.org/10.1097/01. ccm.0000050454.01978.3b 


\section{RESEARCH}

19. Rhodes A, Evans LE, Alhazzani W, et al. Surviving Sepsis Campaign: International guidelines for management of sepsis and septic shock: 2016. Intensive Care Med 2017;43:304-377. https://doi. org/10.1007/s00134-017-4683-6

21. Branch DW. Physiologic adaptations of pregnancy. Am J Reprod Immunol 1992;28(3-4):120-122. https://doi.org/10.1111/j.1600-0897.1992.tb00771.x

20. Oud L. Pregnancy-associated severe sepsis. Curr Opin Obstet Gynecol 2016;28(2):73-78. https://doi org/10.1007/s40121-014-0037-7

Accepted 26 July 2019 